J. Beerten, R. Eriksson, and R. Belmans, "Influence of DC voltage droop on AC system stability," Proc. IET International Conference on AC and DC Power Transmission ACDC 2012, 10th ed., Birmingham, UK, Dec. 4-6, 2012, 5 pages.

Digital Object Identifier: $\underline{10.1049 / \mathrm{cp} .2012 .1960}$

URL (IET Digital Library):

http://digital-library.theiet.org/content/conferences/10.1049/cp.2012.1960

URL (IEEEXplore):

http://ieeexplore.ieee.org/xpl/articleDetails.jsp?arnumber=6521259

(C) 2012 IET. This paper is a postprint of a paper submitted to and accepted for publication in Proc. IET International Conference on AC and DC Power Transmission 2012 and is subject to Institution of Engineering and Technology Copyright. The copy of record is available at IET Digital Library. 


\title{
Influence of DC Voltage Droop Settings on AC System Stability
}

\author{
J. Beerten*, R. Eriksson ${ }^{\dagger}$, R. Belmans* \\ * Department of Electrical Engineering (ESAT), Division ELECTA, University of Leuven (KU Leuven), \\ Kasteelpark Arenberg 10, bus 2445, 3001 Leuven-Heverlee, Belgium, (e-mail: jef.beerten, ronnie.belmans@esat.kuleuven.be) \\ $\dagger$ Electric Power Systems, School of Electrical Engineering, KTH Royal Institute of Technology, \\ Teknikringen 33, 10044 Stockholm, Sweden (e-mail: robert.eriksson@ee.kth.se)
}

Keywords: HVDC transmission, droop control, VSC HVDC.

\begin{abstract}
This paper presents a methodology to study the impact of different DC voltage droop settings on the AC system stability. When several Voltage Source Converters (VSC) in a DC system jointly control the DC voltage by means of droop control, an outage of one converter results in an abrupt change of the active power set-points of several other converters. This relative power change is related to the relative droop settings of the other converters. In this paper, the influence of the power sharing on the AC system stability is discussed by means of a singular value decomposition.
\end{abstract}

\section{Introduction}

The power industry in Europe is facing tremendous challenges. System operators are faced with a system that is operated closer to its limits every year, whereas the construction of new lines is impeded by public opposition. Meanwhile, they face the challenge of connecting significant amounts of variable renewable energy sources, such as offshore wind energy, in the years to come. It is therefore not surprising that the Voltage Source Converter High Voltage Direct Current (VSC HVDC) technology is being installed more and more in the power system, both for connecting offshore wind farms, interconnecting non-synchronised zones or even as an alternative to $\mathrm{AC}$ overhead lines or $\mathrm{AC}$ cables in a meshed AC system. Furthermore, VSC HVDC is the preferred candidate to build multi-terminal HVDC (MTDC) systems, which could be offshore grids or overlaid meshed DC grids [1].

With the plans of building offshore grids or even a European supergrid becoming more and more of a reality, significant research has been conducted on the operation of such meshed DC systems. One of the most prominent research topic is the voltage control of the DC system [2], since the DC voltage plays a vital role, comparable to the frequency in AC systems: Any power deficit that exists in the system as the result of a converter outage results in a fast increase or decrease of the system voltage. Aware of the importance of keeping the voltage close to its nominal value, significant research is being conducted towards joined control strategies using a voltage droop control. In case of a converter outage, all converters involved in the DC voltage control take part of the resulting power deficit, according to their droop characteristics.

The problem assessed in this paper is one that has yet remained unanswered and has not received too much focus so far. It deals with the influence of this DC voltage droop control on the AC power system stability. When a converter outage occurs at one place in the DC system, such a joint voltage droop control will abruptly change the power of the other converters in the system, thereby changing the injections into or withdrawals from the AC systems. As seen from the AC side, this can be considered as a series of contingencies, with abrupt injection or withdrawal changes in the system. This does not only affect the power flows in the AC system, but it can compromise the AC system stability, depending on where the converter outage occurs and where exactly the power deficit is compensated. AC power system stability has been studied by many researchers and in [3] a coordinated control of several HVDC links has been developed to improve the dynamic stability of the AC system. This paper discusses how the DC voltage droop settings and hence the distribution of these abrupt power changes in case of a converter outage influence the AC system stability. An interconnected AC/DC system is considered, with multiple converters connected to different buses of the same AC power system. This means that all power deficits that result from the converter outages have to be redistributed amongst the other converters connected to the same AC system. The paper shows the importance of carefully selecting the DC voltage droop constants and demonstrates how different combinations of DC voltage droop settings evoke different system responses by exciting different system modes. It is shown that different converter outages give rise to other preferred DC voltage droop settings to obtain a suitable distribution of the resulting power deficit.

The paper is organised as follows: Section 2 discusses the DC voltage droop control and the effect of the droop settings on the power sharing. Section 3 introduces the methodology used to analyse the problem, both from a conceptual point-ofview, as well as how to represent the power sharing after an outage. Finally, section 4 analyses simulation results based on the Kundur test system.

\section{DC Voltage Droop Control}

In a DC system, the DC voltage is one of the most crucial system parameters. Any imbalance in active power is directly reflected in a change of the DC voltage at all buses: Any deficit in active 
power results in a rapid decrease and any surplus results in a rapid increase.

The voltage in such a multi-terminal system can either be controlled by one converter or can be spread amongst different converters by using a DC voltage droop control. In case of a converter outage, the voltage in the system deviates from the reference value. In case of a voltage droop control, the power sharing after a converter outage depends on the relative droop values of the different converters.

With a local DC voltage used for droop control, the relation between the active DC power $P_{d c}$ and the voltage $U_{d c}$ at converter $i$ can be written as

$$
P_{d c_{i}}=P_{d c, 0_{i}}-\frac{1}{k_{i}}\left(U_{d c_{i}}-U_{d c, 0_{i}}\right)
$$

with $P_{d c, 0_{i}}$ and $U_{d c, 0_{i}}$ the DC power and voltage reference values and $k_{i}$ the converter droop constant at converter $i$. Alternatively, the DC current can be used for the voltage droop control instead of the active power, as discussed in [4]. Assuming that the entire power imbalance has be redistributed amongst the different converters, the power sharing can be written as a function of the voltage droop constants in the DC grid. This assumption does not take into account the change in system losses after the contingency. Actually, the DC current balance is the one that is maintained: The currents exactly balance each other, whereas the powers depend on the DC voltage in the system.

If a converter is taken out of service the power in this converter is brought down to zero in short time. The other converters in the MTDC system share the power change in order to keep the $\mathrm{DC}$ voltage and thereby the DC power balance. From the AC system point of view these control actions can be seen as abrupt power changes since the time constants of the DC system are much smaller than the ones considered in AC system stability problems. The power change depends on the DC voltage droop settings of each converter except for the disconnected converter which has a fixed change depending on the pre-fault value.

Neglecting the change in DC system losses, an outage of converter $i$ having the steady-state power injection of $p_{i}$ gives rise to the power change in the converters which can be described as follows

$$
\begin{aligned}
& \Delta P_{D C i}=-p_{i}, \\
& \Delta P_{D C j}=p_{i} g_{j}^{\prime},
\end{aligned}
$$

where $g_{j}^{\prime}$ is the modified gain for converter $j$

$$
g_{j}^{\prime}=\frac{g_{j} \Delta U_{d c_{j}}}{\sum_{\substack{k=1 \\ k \neq i}}^{m} g_{k} \Delta U_{d c_{k}}}
$$

with $\Delta U_{d c_{j}}=\left(U_{d c_{i}}-U_{d c, 0_{i}}\right)$ and the converter gain $g_{j}$ equal to $k_{j}^{-1}$.

It can be observed from these equations that the actual redistribution of the active power depends on the DC grid voltage profile after the fault, which impedes a straightforward analysis.
In case of a common voltage feedback signal used by all converters, as proposed in [5], the droop equation simplifies to

$$
P_{d c_{i}}=P_{d c, 0_{i}}-\frac{1}{k_{i}}\left(U_{d c}^{+}-U_{d c, 0}^{+}\right)
$$

with $U_{d c}{ }^{+}$the common converter feedback signal and $U_{d c, 0}{ }^{+}$its reference value. It can easily be observed that with a common voltage feedback, the relative power $g_{j}^{\prime}$ of converter $j$ after a contingency can be written as

$$
g_{j}^{\prime}=\frac{g_{j}}{\sum_{\substack{k=1 \\ k \neq i}}^{m} g_{k}},
$$

thereby no longer depending on the system state after the contingency. In this implementation, it becomes possible to link the power sharing duties to the droop settings of the converters. In case of a local voltage feedback, the link between droop settings and power sharing is less apparent because of the influence of the DC voltages. The disadvantage with using a common voltage reference, however, is the need for a communication system.

In this paper, the DC grid itself is left out of the analysis and it is assumed that no converter current limits are hit as a result of the voltage droop control. The first approximation comes down to the situation in which a common reference voltage is used or in which the voltage drops in (4) are comparable for all buses in the system, hence approximating a copper plate.

\section{Methodology}

This section discusses the methodology used to study the effect of converter outages on the AC system. The main focus of this work is on the adverse effect of a converter outage on the AC system stability. The DC grid dynamics are left out of the analysis by assuming that the power changes resulting from both the converter outage, as well as the ones resulting from the droop control action take place instantaneously. This assumption is justified by the fact that the AC grid dynamics under consideration are an order of magnitude slower than the ones from the DC system. The first part of this section focuses on the multi-input multi-output (MIMO) System Analysis, the second part specifically addresses the converter outage analysis.

\subsection{MIMO System Analysis}

A converter outage and the subsequent power changes of the voltage droop controlled converters can be regarded as a change of different system inputs and hence a disturbance in a certain direction, depending on the droop settings. Consequentially this disturbance impacts different system variables or a combination thereof. It is known that MIMO control systems pose complexity in issues as gain, phase and directions which are strongly interrelated. In MIMO systems, the magnitude of the output signal depends not only on the magnitude of disturbances, but also on the relative phase displacement between the disturbance 
signals i.e. the disturbance directions. Thus, the gains have an impact of the adverse effect in the AC system due to a converter outage. One way of looking at the directionality and system gain is to linearise the system around the operating point and perform singular value decomposition (SVD). Modelling the $\mathrm{AC} / \mathrm{DC}$ system around an equilibrium point by a linear system

$$
G_{d}(s)=\begin{array}{l|l}
A & B \\
\hline C & D
\end{array},
$$

with matrices $A, B, C$ and $D$ defining the state-space representation of the linearised system. Disturbances on the DC side may be modelled by disturbance models $G_{d}(s)$ having the active power injection into the AC system as the input signals and the speed deviation of the generators as outputs. The disturbance response highly depends on the voltage droop settings since these settings, in combination with the converter outage $i$, decide the direction $v_{d_{i}}$ in the disturbance channel.

SVD is associated with the principal directions and gains. The maximum $\bar{\sigma}\left(G_{d}(s)\right)$ and minimum $\underline{\sigma}\left(G_{d}(s)\right)$ singular values are given by [6]

$$
\underline{\sigma}\left(G_{d}(\jmath \omega)\right) \leq \frac{\left\|G_{d}(\jmath \omega) v_{d}\right\|_{2}}{\left\|v_{d}\right\|_{2}} \leq \bar{\sigma}\left(G_{d}(\jmath \omega)\right),
$$

with

$$
\begin{aligned}
& G_{d}(\jmath \omega) \bar{v}=\bar{\sigma} \bar{u}, \\
& G_{d}(\jmath \omega) \underline{v}=\underline{\sigma} \underline{u} .
\end{aligned}
$$

The vector $v_{d}$ is an arbitrary input to the system, $\bar{v}$ corresponds to the input direction with largest amplification, and $\bar{u}$ is the corresponding output direction in which the inputs are most effective. The least effective input direction is associated with $\underline{v}$ corresponding to the output $\underline{u}$. To minimise the impact, these directions should be considered when setting the voltage droop gains in the converters.

\subsection{Converter Outage Analysis}

As the relation between the converter gains is of concern, the entire subset of gains has to be scaled to achieve an acceptable dynamic response [7]. In this analysis, we disregard the intermediate dynamics and we model the outage as if the converter powers change abruptly at the same time. Therefore, only the relative values of the converter gains are of concern for this analysis. Hence, with the power sharing after an outage of converter $i$ as in (6), an additional equation is defined such that

$$
\sum_{k=1}^{m} g_{k}=1
$$

The disturbance caused by the outage of converter $i$ can be expressed mathematically as

$$
v_{d_{i}}=p_{i}\left[g_{1}^{\prime}, \ldots, g_{i-1}^{\prime},-1, g_{i+1}^{\prime}, \ldots, g_{m}^{\prime}\right]^{T}
$$

The input signal for each converter outage $v_{i}$ can be rewritten as a linear combination of the input directions $V=[\underline{v} \ldots \bar{v}]$ associated with the singular values

$$
v_{d_{i}}=V \alpha_{d_{i}} .
$$

By using a singular value decomposition of the linearised matrix $G_{d}$ from (7), the system response or gain for $v_{d_{i}}$, the input signal associated with the outage of converter $i$, can be rewritten as

$$
\left\|G_{d} v_{d_{i}}\right\|_{2}=\left\|\Sigma^{T} \alpha_{i}\right\|_{2}
$$

with $\Sigma=[\underline{\sigma} \ldots \bar{\sigma}]^{T}$. The system response is studied in the frequency domain to observe maximum and minimum gains at different frequencies.

The abrupt power changes in the different converters are modelled as step functions. In the Laplace s-domain the step or heaviside function is $1 / s$, thus, it has a wide frequency spectrum. This means that a converter outage can excite all modes to some extent.

The frequencies where the gain peaks should be considered when minimising the adverse effect of a converter outage. A singular value plot provides a means to generalise this information by generating a plot of the frequency dependence of singular values of the transfer matrix evaluated at different frequencies. This plot indicates how the modes can be excited by the MTDC system. The aim in this study is to minimise the disturbance gain for the lowest damped mode or, put differently, to reduce the excitation of these modes during an outage. The total gain $\beta_{\text {tot }}$, including all possible converter outages, can be expressed as

$$
\beta_{t o t}=\sqrt{\sum_{\forall i}\left\|G_{d} v_{d_{i}}\right\|_{2}^{2}}
$$

The aim of the optimisation is thus to minimise the gain for all converter outages with respect to the DC voltage droop gains. This can be formulated as

$$
\text { with } \sum_{k=1}^{m} g_{k}=1 \text { and } g_{k} \geq 0 .
$$

and by solving this problem the adverse effect is minimised.

\section{Simulation Results}

To verify the developed methodology, simulations are performed in the Kundur test system [8] in which an MTDC system is connected and transferring $200 \mathrm{MW}$ as shown in Fig. 1.

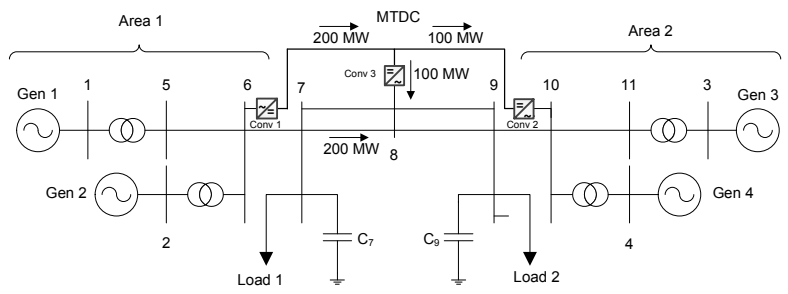

Figure 1. Two-area four-machine system with an MTDC system.

After linearising the system, one inter-area mode and two local modes are found. Table 1 contains the eigenvalues and as can be seen the modes are positively damped. 


\begin{tabular}{|c|c|c|c|}
\hline Eigenvalue & Damping & Frequency & Mode shape \\
\hline$-0.0999 \pm \mathrm{j} 3.3424$ & $2.99 \%$ & $0.532 \mathrm{~Hz}$ & G1,G2-G3,G4 \\
$-0.5079 \pm \mathrm{j} 6.2127$ & $8.15 \%$ & $0.988 \mathrm{~Hz}$ & G3-G4 \\
$-0.5156 \pm \mathrm{j} 5.8188$ & $8.83 \%$ & $0.926 \mathrm{~Hz}$ & $\mathrm{G} 1-\mathrm{G} 2$ \\
\hline
\end{tabular}

Table 1. Modes of the test system

To further analyse the system we study the SVD plot, shown in Fig. 2. The gain for the inter-area mode is much higher than for the local modes. The DC voltage droop gains need to be optimised to minimise the gain for the inter-area mode in case of a converter outage.

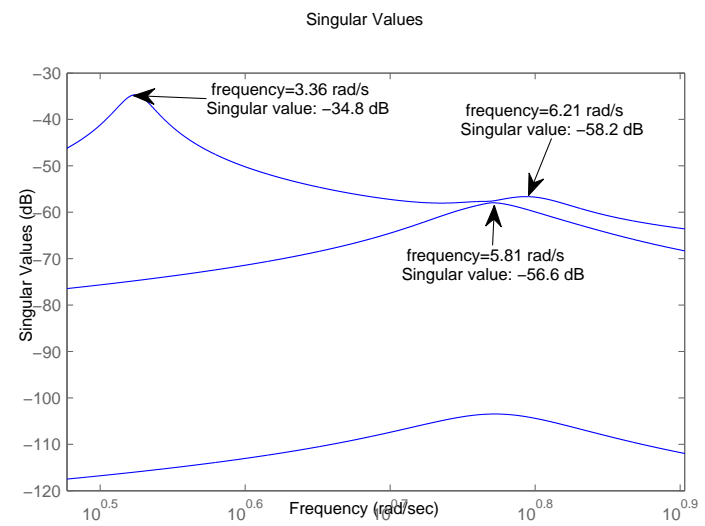

Figure 2. SVD plot for the test system

In this system there are three converters so there are three possible converter outages. For each converter outage we may vary the gains and plot the gain. Evaluating the SVD at the frequency of the inter-area mode and using (14) and (13) the system gain can be plotted for different distribution of the DC voltage droop gain. $g_{1}$ and $g_{2}$ are along the x-axis and y-axis, $g_{3}$ is a function of $g_{1}$ and $g_{2}$ as follows

$$
\begin{aligned}
& g_{1}=0 \longrightarrow 1, \\
& g_{2}=0 \longrightarrow 1-g_{1}, \\
& g_{3}=1-g_{1}-g_{2} .
\end{aligned}
$$

Fig. 3 shows the disturbance gain for an outage in converter 1 . Clearly, the DC voltage droop gain $g_{1}$ has no impact on the disturbance gain since the corresponding converter is out of service. In the $g_{2}$ direction the system gain increases. Intuitively, we expect larger impact when the power imbalance is dealt with more locally by converter 3 instead of converter 2 . This is confirmed by the results.

Fig. 4 displays the system gain for converter outage 2 . In this case it is also expected that the disturbance gain is lower if converter 3 solves the power mismatch. As can be seen in the figure the result is similar to the previous case.

The last case considered is when converter 3 has an outage. In this case the DC voltage gain $g_{3}$ has no impact on the disturbance gain. The disturbance gain is low if the power imbalance is equally distributed between the converters 1 and 2 as shown in Fig. 5.

In Fig. 6 the total disturbance gain is shown as given in (15).

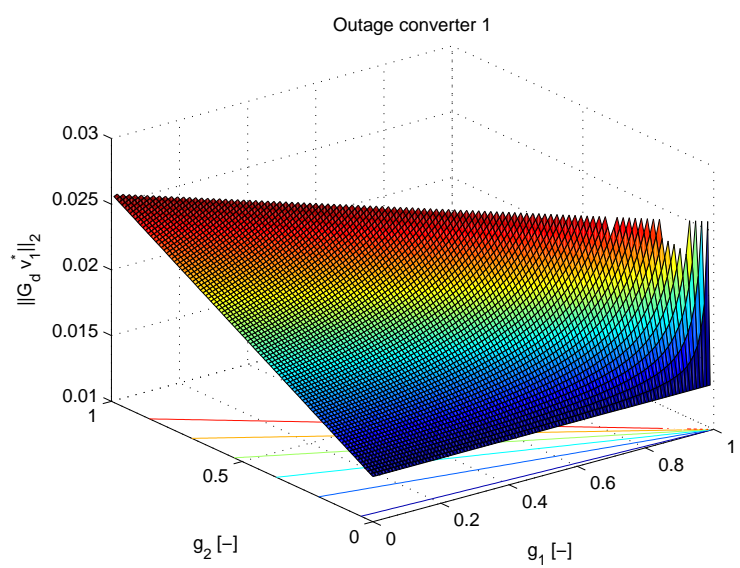

Figure 3. System gain for an outage in converter 1.

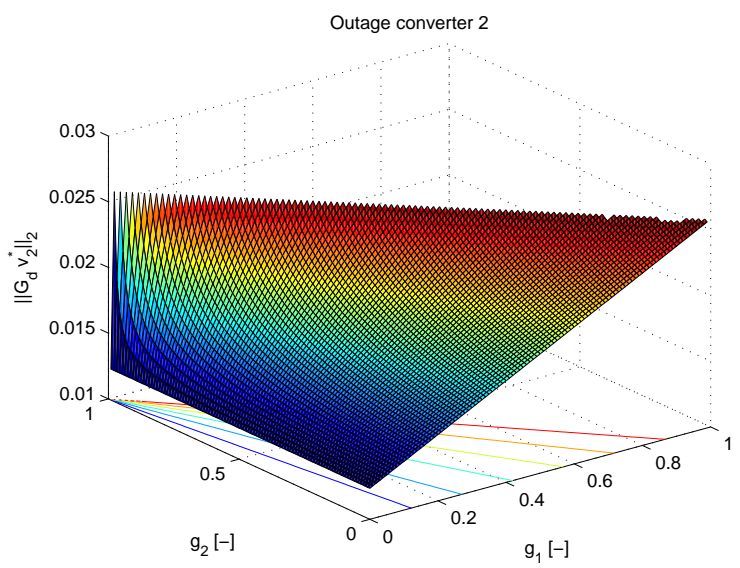

Figure 4. System gain for an outage in converter 2.

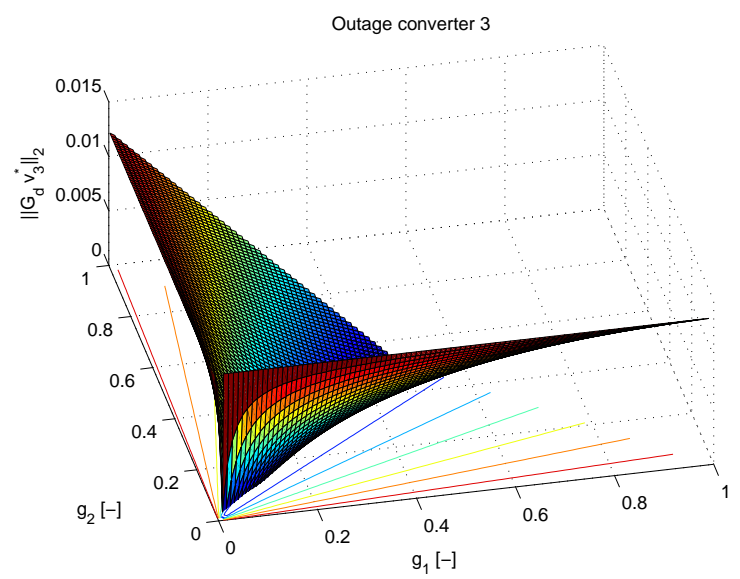

Figure 5. System gain for an outage in converter 3.

It shows that for this system, the better option is to have a higher DC voltage droop gain in converter 3 .

To verify the result, time simulations have been performed using Power System Analysis Toolbox (PSAT), a Matlab toolbox for electric power system analysis and simulation [9]. An outage of converter 1 has been considered, both for a normalised set of gains $g=\left[\begin{array}{llll}1 / 3 & 1 / 3 & 1 / 3\end{array}\right]$ and $g=\left[\begin{array}{lll}1 / 6 & 1 / 6 & 2 / 3\end{array}\right]$. It can 


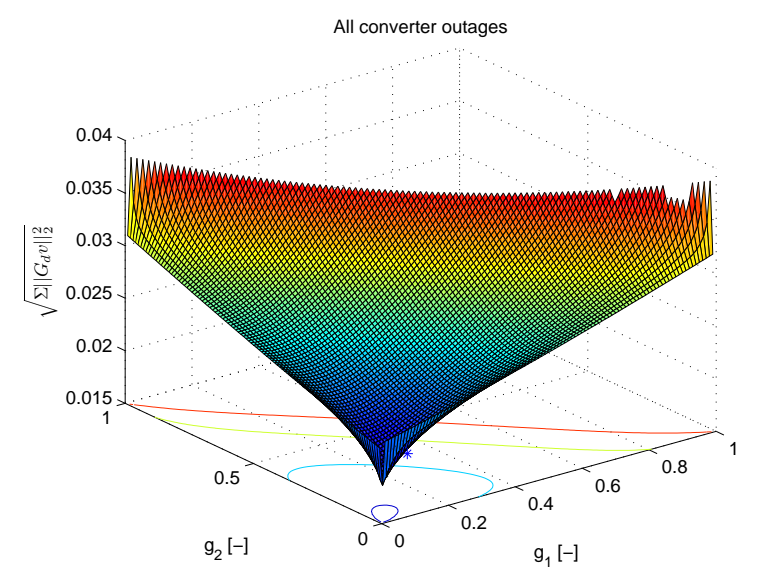

Figure 6. Total gain

be expected that the second set of gains should result in an overall lower system impact. The resulting generator angles are shown in Fig. 7. The angle deviation gives a better visualisation than the speed signals. From these results, the analysis results

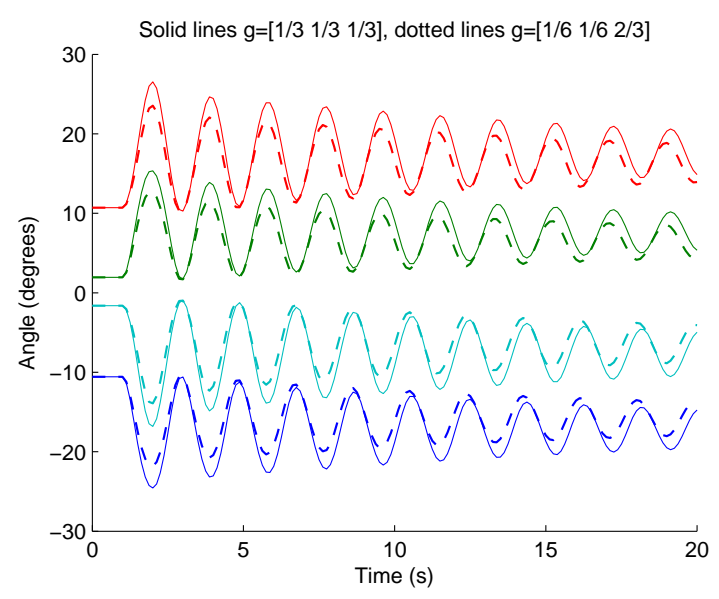

Figure 7. Time simulations for an outage of converter 1

from the previous section are confirmed: It is beneficial for the $\mathrm{AC}$ system to have a higher share of the power imbalance in converter 3 to lower the impact of a converter outage.

\section{Conclusion}

In this paper, a methodology has been presented to assess the impact of DC system contingencies on the AC power system. The methodology, which is based on MIMO system analysis and SVD, allows to detect the input directions that cause the smallest effect on the system outputs due to disturbances on the DC side. The contribution of this paper is a method which derives the voltage droop settings to minimise the adverse effect of a disturbance on the DC side. Simulation results show the validity of the proposed approach.

\section{Acknowledgement}

Jef Beerten is funded by a research grant from the Research Foundation - Flanders (FWO).

Robert Eriksson is support by the Centre of Excellence in Electrical Engineering $\mathrm{EKC}^{2}$ at $\mathrm{KTH}$.

\section{References}

[1] D. Van Hertem and M. Ghandhari, "Multi-terminal VSC HVDC for the European supergrid: Obstacles," Renewable and Sustainable Energy Reviews, vol. 14, no. 9, pp. 31563163, Dec. 2010.

[2] T. Haileselassie, K. Uhlen, and T. Undeland, "Control of multiterminal HVDC transmission for offshore wind energy," in Proc. Nordic Wind Power Conference Presentation, Rønne, Denmark, Sep. 10-11, 2009.

[3] R. Eriksson, "Coordinated control of HVDC links in transmission systems," Ph.D. dissertation, KTH Royal Institute of Technology, Stockholm, Sweden, 2011.

[4] J. Beerten and R. Belmans, "Modeling and control of multiterminal VSC HVDC systems," Energy Procedia, vol. 24, pp. 123 - 130, 2012.

[5] C. Barker and R. Whitehouse, "Autonomous converter control in a multi-terminal HVDC system," in Proc. IET ACDC '10, London, UK, Oct. 20-21, 2010.

[6] S. Skogestad and I. Postlethwaite, Multivariable Feedback Control, Analysis and Design. Wiley, 2001.

[7] E. Prieto-Araujo, F. D. Bianchi, A. Junyent-Ferre, and O. Gomis-Bellmunt, "Methodology for droop control dynamic analysis of multiterminal VSC-HVDC grids for offshore wind farms," IEEE Trans. Power Del., vol. 26, no. 4, pp. 2476-2485, 2011.

[8] P. Kundur, Power System Stability and Control. McGrawHill Inc, New York, 1993.

[9] F. Milano, "An open source power system analysis toolbox," IEEE Trans. Power Syst., vol. 20, no. 3, pp. 1199 - 1206, aug. 2005. 